\title{
Severe graphene nanoplatelets aggregation as building block for the preparation of negative temperature coefficient and healable silicone rubber composites
}

\author{
L. Valentini ${ }^{\text {a, }}{ }^{*}$, S. Bittolo Bon ${ }^{a}$, N.M. Pugno ${ }^{b, c, d,{ }^{* *}}$ \\ a Dipartimento di Ingegneria Civile e Ambientale, Universita di Perugia, UdR INSTM, Strada di Pentima 4, 05100 Terni, \\ Italy \\ ${ }^{b}$ Laboratory of Bio-Inspired and Graphene Nanomechanics, Department of Civil, Environmental and Mechanical \\ Engineering, University of Trento, Trento, Italy \\ c Center for Materials and Microsystems, Fondazione Bruno Kessler, Trento, Italy \\ a School of Engineering and Materials Science, Queen Mary Univerisity of London, Mile End Road, London, United \\ Kingdom
}

\begin{abstract}
With the request of higher performance in automotive products, sealing components and materials resisting to severe conditions, the performance requirements for silicones are becoming ever more diverse and sophisticated. In this article we prepared silicone rubber (SR)-graphene nanoplatelets (GNPs) composite via liquid mixing method; the mechanical strength of the GNPs estimated by applying quantized fracture mechanics suggested a severe GNPs agglomeration that was confirmed by scanning electron microscopy analysis. We observe that such SR/GNP composite behaves as a negative temperature coefficient material, exhibiting electrical resistance decrease with temperature increase. It was also shown how the damaged SR/GNP composite can be healed by simple thermal annealing. The healing mechanism was rationalized in terms of "living" reactive species that are not consumed by curing at room temperature and promote, when thermally activated, the crosslinking among the damaged network of oligomers. The healing efficiency, expressed as crack length vs. annealing temperature, has been estimated again applying the principles of quantized fracture mechanics. These results could satisfy many of the demanding requirements of the silicone rubber materials used in daily life and indicate that SR/GNP composites can act as healable and temperature sensor materials e. g. for seals, hoses and automotive sector.
\end{abstract}

\section{Introduction}

Rubber materials are commonly considered the workhorse of the industrial and automotive rubber products industries [1e3]. As a significant candidate of them, the silicone rubber (SR) offers a unique combination of chemical and mechanical properties that organic elastomers cannot match. Silicone rubber withstands high and low temperatures far better than organic rubbers. Silicone rubber can be used indefinitely at $150 \mathrm{C}$ with almost no changes in its properties. It withstands use even at $200 \mathrm{C}$ and some products can withstand heat of $350 \mathrm{C}$ for short periods. Silicone rubbers are thus suitable as a material for rubber components used in high temperatures environments. These properties make silicone rubber the most used material for applications in extreme temperature conditions due to its good thermal stability and especially excellent elasticity [4,5]. Moreover, such soft and flexible materials have attracted attention due to their potential applications in advanced strain sensors [6e8].

Silicone rubbers are usually reinforced with carbon black, carbon nanotubes and graphene nanoplatelets to obtain improvements in thermal and electrical properties [9e11]. Compared with the traditionally used reinforcing fillers like silica, graphene nanoplatelets (GNPs) have recently attracted attention as a negative temperature coefficient material [12], exhibiting rapid electrical resistance decrease with temperature increase. Kong et al. [12] demonstrated how the electrical resistance is size dependent, decreasing with increasing the graphene thickness. These findings could suggest that agglomeration can be a viable method to produce negative temperature coefficient materials. It 
was also found that GNPs were similar to conventional negative temperature coefficient materials and this finding suggests the potential use of GNP based composites for temperature sensors in rubber made automotive components.

Elastomeric applications are also susceptible to mechanical and chemical damage (e.g. scratches, cuts and punctures). Such damages result in the loss of the originally intended functions of the elastomers leading to spillage, contamination, safety hazards or just lost of performance. Such damages are particularly problematic when the elastomers are used as seals, hoses and coatings. After the occurrence of the first cracks or surface damage, the material is especially susceptible to further damage. For this reason, seals, hoses or coatings have to be frequently checked and mended or even replaced with the subsequent cost. The development of elastomers with self-healing properties, i.e. the realization of structures able to repair mechanical damage, is an important challenge from industrial point of view for the development of polymeric materials that have much greater lifespans than currently available.

For decades, the scientific community focused the attention to developing self-healing polymeric materials to improve the safety and lifetime [13]; the storage of healing agents in the materials that are released upon damage is the most used approach. This technology generally consists in the exploitation of microcapsules [14e16] which store the healing agents into the polymer matrix. The healing agent is released from the crack to then restore it. The main drawbacks of such approach consist in the possibility to heal the material only once at the same location and the reduction of mechanical properties due to the inclusion of microcapsules in the polymer matrix that behaves as defects. Some outstanding results to overcome the utilization of microcapsules came from Leibler et al. [17] that used a mixture of fatty diacid and triacid condensed with diethylene triamine and then reacted with urea giving a crosslinked rubber that in contrast to conventional cross-linked rubbers made of macromolecules, when cut, can be repaired by bringing together fractured surfaces to selfheal at room temperature. A step forward toward silicone based elastomers has been obtained recently by Bao et al. [18] who synthesized a network of poly(- dimethylsiloxane) polymer chains crosslinked by coordination complexes that combines high stretchability and autonomous selfhealing. The most common silicones are the polydimethylsiloxanes, trimethylsilyloxy terminated, with the following structure $\mathrm{Me} 3 \mathrm{SiO}(\mathrm{SiMe} 2 \mathrm{O}) \mathrm{nSiMe} 3$ and many of such silicone polymer systems involve the reaction of a silane-hydrogen $(\mathrm{SiH})$ component with a silanol $(\mathrm{SiOH})$ component. Thus the presence of free silanol groups can be used to catalyse the healing of such polymers when activated by temperature. For example, very recently it was shown [19] that silicone-based sealants exposed to a hydrocarbon flame, can be easily healed. In this case healing did not require the delivery of coordination complexes $[18,19]$.

As stated by the above recent works the research on self-healing organic polymers has grown recently, but one simple self-healing mechanism from more than 60 years ago has been nearly forgotten until Zheng et al. [20] have demonstrated that silicone rubber that has been cut in half can completely repair itself through heat-activated reversible bonding. They showed that the healed interface had strength comparable to the cohesive strength of the undamaged elastomer. They obtained a completely self-healed silicon rubber at $90 \mathrm{C}$ for $24 \mathrm{~h}$. The temperature thus is a crucial parameter that can be used to heal silicone rubbers and a viable method could be the addition of thermally conductive inclusions into the polymer matrix for a better heat transfer.

Because thermal conductivity of elastomers is very low, the heat build-up is harmful to elastomers, because elastomers are susceptible to thermal degradation [21,22]. Composites with carbonbased fillers showed thermal stability, light weight, and high thermal conductivity $[23,24]$.

These properties prompt us to believe that integrating GNPs with SR with free silanol groups might generate a novel healable composite material, which deals self-repairing by thermal annealing and temperature activation of the electrical conductivity. Our material paves potential applications in sealing and temperature sensors of automotive components. 


\section{Experimental details}

Liquid rubber (GLS-50 purchased from PROCHIMA®) was used for casting with a cold cure by polycondensation. Before using, we add to the rubber $5 \%$ of T30 catalyst (purchased from PROCHIMA®). The complete vulcanization takes $18 \mathrm{e} 20 \mathrm{~h}$ at room temperature. To accelerate this process, the blend was put in a warm place $(30 \mathrm{C})$, but the reaction was too fast and did not allow the escape of air bubbles.

GNPs were purchased from Cheaptubes (bulk density $0.04 \mathrm{~g} / \mathrm{cm} 3$, thickness $8 \mathrm{e} 15 \mathrm{~nm}$, lateral dimension about $1.5 \mathrm{~mm}$ ). GNPs were dispersed in liquid silicone rubber (1\%wt.) through the utilization of a Dispermat (500 rpm for $1 \mathrm{~h}$ ) to facilitate the dispersion of GNPs. Then the catalyst was added. The liquid composite was deposited onto a silicone mould and the vulcanization was obtained in $18 \mathrm{e} 20 \mathrm{~h}$ at room temperature.

The melting behaviours of the samples were tested by differential scanning calorimeter (DSC) using a TA Q200 DSC analyser under nitrogen atmosphere. Samples were heated from $80 \mathrm{C}$ to $150 \mathrm{C}$, cooled to $80 \mathrm{C}$, and heated to $150 \mathrm{C}$ again. In all heating and cooling cases, the rate was set at 10 $\mathrm{C}$ min-1. Thermogravimetric analysis (TGA) were performed in nitrogen with a TG/DTA Extar 6300 at a heating rate of $10 \mathrm{C}$ min1. Field emission scanning microscopy (FESEM) was used to investigate the cross section of the samples obtained by fracture in liquid nitrogen.

Neat silicon rubber (SR) and silicon rubber added with GNP (SR/ GNP) were characterized with Fourier transform infrared spectroscopy (FTIR) to verify the occurrence of the polymerization and the effects induced by the GNP presence. The measurements were realized using a JASCO FT/IR615 spectrometer, in the $500 \mathrm{e} 4000 \mathrm{~cm} 1$ range transmission mode.

The tests were performed on a thin film of the SR and of the SR/ GNP: the reactive mixtures consisting of neat SR, SR/GNPs and T30 catalyst were deposited onto silicon substrates and the measurements were recorded before and after the polymerization of the samples that was performed at room temperature for $24 \mathrm{~h}$. A substrate of neat silicon was used to subtract the background spectrum.

$\mathrm{SR}$ and SR/GNPs polymerized samples were then post annealed in an oven at $150 \mathrm{C}$ and at $250 \mathrm{C}$ for $2 \mathrm{~h}$ and FTIR spectra were recorded to investigate the modifications induced by the annealing to their chemical structure.

The electrical characterization of both neat SR and SR/GNP composite was performed, by using a computer controlled Keithley 4200 source measure unit. The electrical current was recorded by biasing the samples at $50 \mathrm{~V}$ and $100 \mathrm{~V}$ at different temperatures.

\begin{tabular}{llllllll} 
The samples & were & cut & into & strips & of & $\sim 100$ & $\mathrm{~mm}$ \\
\hline
\end{tabular}

$2.5 \mathrm{~mm}$. Before healing, a $3 \mathrm{~mm}$ cut was made in the middle of the sample along the strip traverse direction, and then the cut sample was healed by thermal annealing in an oven up to $250 \mathrm{C}$ for $2 \mathrm{hr}$. The mechanical properties were measured by a universal tensile testing machine (Lloyd Instr. LR30K) with a $250 \mathrm{~N}$ load cell at room 12 temperature. The extension rate was $50 \mathrm{~mm}$

$\min 1$ and the gauge length was $50 \mathrm{~mm}$.

\section{Results and discussion}


The thermal properties of neat SR and SR/GNP composites were investigated. Fig. 1(a) and (b) show the TGA curves and the corresponding differential thermogravimetric (DTG) analysis curves. Fig. 1b showed that both samples decomposed with a one-step process, which meant the GNPs did not break the network of the SR. The incorporation of the GNPs did not change the onset temperature of the composites compared to that of the neat SR. DSC analysis for the SR and the SR/GNP composites was then performed and the results were shown in Fig. 1c. It was known from the literature [25] that the glass transition temperature of the SR was lower than $100 \mathrm{C}$, so the endothermic peak observed in Fig. 1c corresponds to the melting temperature. It was found that the presence of the nanofiller did not affect the melting temperature of the neat SR matrix.
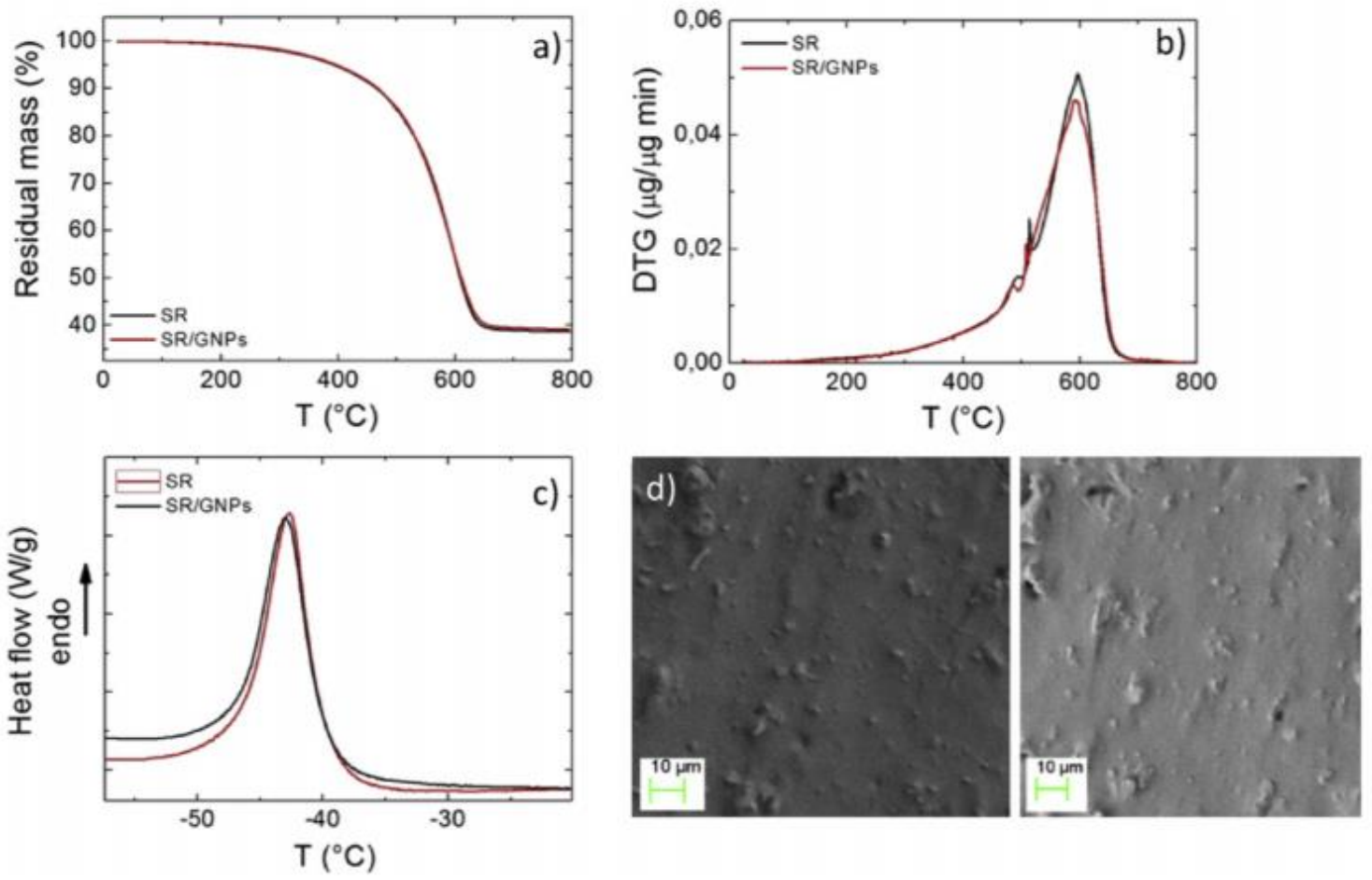

Fig. 1. (a) TGA curves and corresponding (b) DTG curves of neat SR and SR/GNP composite. (c) DSC curves of neat SR and SR/GNP composite. (d) FESEM images of the cross sections of neat SR (left panel) and SR/GNP composite (right panel).

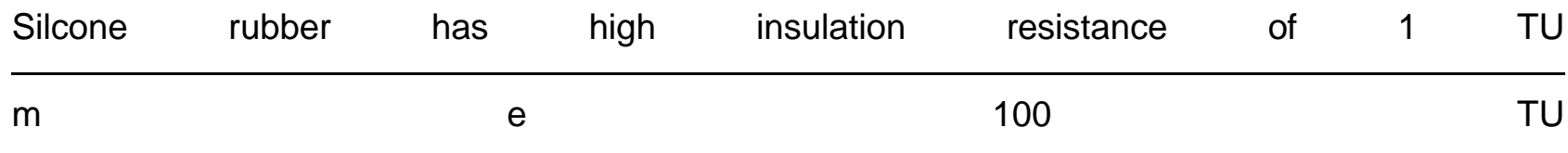

$\mathrm{m}$, and its insulating properties are stable over a wide range of temperature as confirmed by the data reported in Fig. 2a where no change of the electrical conductivity was detected biasing the sample with $50 \mathrm{~V}$ up to $250 \mathrm{C}$.

Fig. $2 \mathrm{~b}$ shows that the electrical conductivity (electrical resistance) of the SR/GNP composite increased (decreased) with temperature. This effect of the temperature is similar to what has been observed on the GNP resistance by Kong et al. [12] and Zhuge et al. [26]. Kong et al. [12] used the following equation to model the negative temperature coefficient behaviour

$$
\mathrm{R}_{\mathrm{T}}=\mathrm{R}_{0} \exp \left(\mathrm{B} *\left(\mathrm{~T}_{0}-\mathrm{T}\right) / \mathrm{T}_{0} \mathrm{~T}\right)
$$

where RT is the electrical resistance as function of temperature $T, B$ is a material characteristic temperature, and $\mathrm{R} 0$ is the resistance at room temperature (e. g. T0 1/4 $298 \mathrm{~K}$ ). From the data of Fig. $2 \mathrm{~b}$, in the temperature range between $298 \mathrm{~K}$ and $523 \mathrm{~K}, \mathrm{~B}$ was determined to be $3393 \mathrm{~K}$ in the respective resistance range of 
$109 \mathrm{U}$. This $\mathrm{B}$ value is close to that of the conventional metal oxide negative temperature coefficient materials, typically in the range of $2000 \mathrm{e} 5000 \mathrm{~K}$ [27].
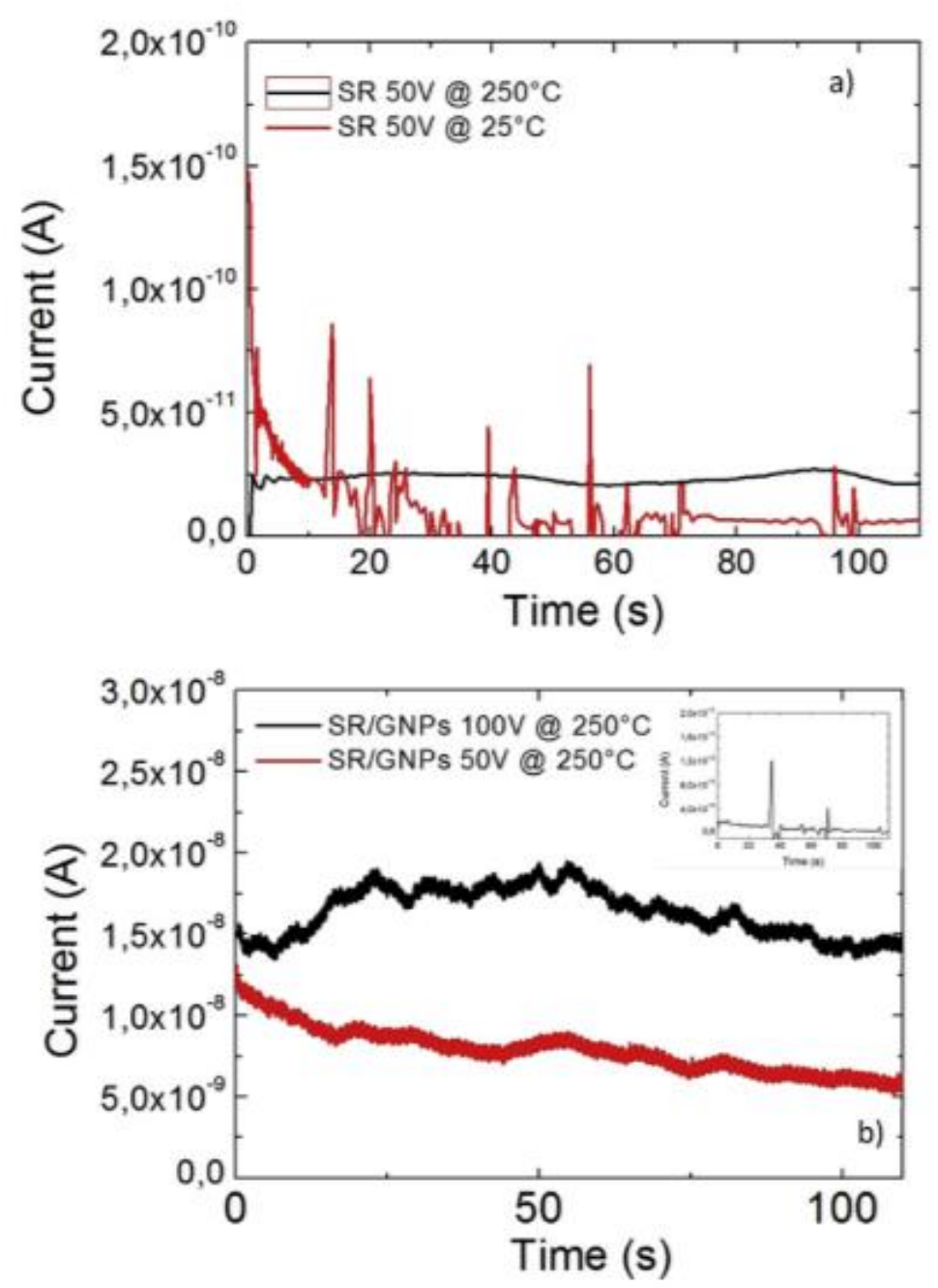

Fig. 2. Current-time data of (a) SR and (b) SR/GNP composite recorded at different bias voltages and temperatures. The inset of panel (b) shows the current-time data of SR/GNP composite biased with $50 \mathrm{~V}$ at room temperature.

The obtained results thus can be explained in the following way: the high resistivity of GNPs at room temperature leads to small current flowing through the composite and therefore, the conduction is low. As the temperature increases, the resistivity of GNPs decreases; thus the current flows through the sample.

The slight decrease of the conductivity vs. time observed in Fig. $2 b$ is due to a self-heating mechanism; by decreasing the resistance, the flow of the current is higher and the sample heats by Joule effect with time. Considering that the thermal expansion coefficient of silicone rubber is $2.0 \mathrm{e} 2.5$ times higher than other organic rubber, such thermal expansion leads to an increase of gap distance between the GNP inclusions, resulting in a slight decrease in electrical conductivity.

The mechanical performance of the pristine SR and the SR/ GNP composites was then tested in terms of typical strainestress behaviour with the curves shown in Fig. 3(a) and (b). The values of fracture strength (i.e. sF, the maximum of the stress-strain curves), the elongation at break (i.e. EB, the ultimate strain) and the toughness (i.e. the area underlying the stress-strain curves) are summarized in Fig. 3(c) and (d). The mechanical performance of the SR/GNPs composite had an 
improvement of the tensile strength while the elongation at break decreased maintaining almost the same toughness. The increasing of the tensile strength of the nanocomposites indicated that GNPs and SR had interfacial adhesions, and the GNPs deserve to transfer the tensile load when the nanocomposite was stretched.

We rationalized the test on the healed samples by Quantized Fracture Mechanics (QFM) theory [28]. For an infinite sheet with a central crack of length, $2 a$, subjected to a uniform stress $s$ the fracture toughness is given by $\mathrm{KIC} 1 / 4 \mathrm{SF}(\mathrm{a})[\mathrm{p}(\mathrm{a} p q / 2)] 1 / 2$ where $a$ is the crack half-length, $q$ the fracture quantum, and $\mathrm{SF}$ (a) the fracture strength, with the latter for vanishing crack length that can be expressed as the combination of the strength of the SR matrix (sF,SR) and the strength of the GNPs (sF,GNPs):

$$
\sigma_{\mathrm{F}}(a)=f \sigma_{\mathrm{F}, \mathrm{GNPS}}+(1-f) \sigma_{\mathrm{F}, \mathrm{SR}}
$$

whereas according to QFM the notched and unnotched strengths are related via:

$$
\sigma_{F}(a)=\sigma_{F}(a=0)[1+2 \pi \cdot a / q]^{-1 / 2}
$$

where $f$ is the volume fraction of the GNPs. From Eq. (2a) we estimate an equivalent strength of graphene nanoplatelets $\mathrm{SF}, \mathrm{GNPs} 1 / 443 \mathrm{MPa}$, thus suggesting agglomeration of GNP in the current composite. Note that for good GNPs dispersion with low agglomeration in rubber composites, we recently found for SF,GNPs a value of about $800 \mathrm{MPa}$ [29].

Schematically for a composite formed by a matrix and a single phase material (i. e. GNPs), the agglomeration of the single phase is defined by the number $n$ of nano-inclusions in a single agglomeration. Assuming a Weibull distribution, the mechanical resistance of the single agglomeration, sF,GNPs, rescales, with Weibull exponent $a$, with its size $\mathrm{n}$ and the number of adjacent vacancy defects $\mathrm{m}[30]$, as it follows:

$$
\sigma_{\mathrm{F}, \mathrm{GNPS}} \cong \sigma_{1} /\left((1+\mathrm{m})^{0.5} \times \mathrm{n}^{(1 / \alpha)}\right)
$$

where fracture mechanics would predict a $1 / 42$ for $2 \mathrm{D}$ inclusions and $\mathrm{s} 1$ ( 70GPa) is the strength of a single layer graphene [30]. Measuring a thickness of about $6 \mathrm{~mm}$ for the single GNP inclusion (see Fig. 4a) and assuming an average stacking layer distance of about $0.35 \mathrm{~nm}$ [29] ( $\sim$ 17000), we obtain a value of $m \sim 150$.

Assuming $q=0$ according to classical Linear Elastic Fracture Mechanics (LEFM) theory, the fracture toughness value calculated for both set of prepared samples is $0.0280 \mathrm{MPa}$ m1/2 for SR/GNPs and $0.0348 \mathrm{MPa} \mathrm{m} 1 / 2$ for SR. According to Eq. (2b), knowing the fracture strength for undamaged and $3 \mathrm{~mm}$ cut sample, yields qSR $1 / 43.1 \mathrm{~mm}$ and qSR/GNPs $1 / 40.9 \mathrm{~mm}$. The effect of the thermal annealing on the crack length reduction has been reported in Fig. 4b. The healed crack length is determined from Eq. (1) knowing the values of the fracture quantum and the fracture strength of the sample. From this figure it is clear that the annealing up to $250 \mathrm{C}$ did not allow the repairing of the initial crack of the SR sample while the composite was almost totally healed, being about $95 \%$ the ratio between the healed crack and initial crack length. Optical images confirmed the healing of the $\mathrm{SR} / \mathrm{GNP}$ composite. From the comparison of the two images it is evident that the crack in the SR/ GNPs sample almost disappeared after annealing. After the annealing, the composite showed a healing efficiency of the tensile strength that was about $87 \%$ as reported in Fig. $3 \mathrm{~d}$. Computed values of the healed crack length according to QFM are reported in Table 1.

The chemistry of this self-healing composite deserves a discussion. The mechanism of self-healing in our silicone composite can be rationalized if we consider a material that contains reversible bonds that can be thermally activated subsequent to damage. Such material involves association reactions 
that happen after the polymerization event. This system is designed so that damage can be healed thanks to kinetic reactions in solid polymers at temperatures requires activation of the damaged area with heat $[19,20,31]$. Catalyst is used to catalyse the silicone polymerization as sketched in the inset of Fig. 5a where free silanol $(\mathrm{SiOH})$ groups attach to form the siloxane (-Si-O-Si-) chain. Thus free $\mathrm{SiOH}$ silanol groups if not exhausted can be reactivated by temperature to complete the curing of the system by crosslinking reaction. The involving in the reaction of a sylane-hydrogen $(\mathrm{SiH})$ group with a silanol group resulting in the liberation of water, requires the investigation by spectroscopy of the hydroxyl region.
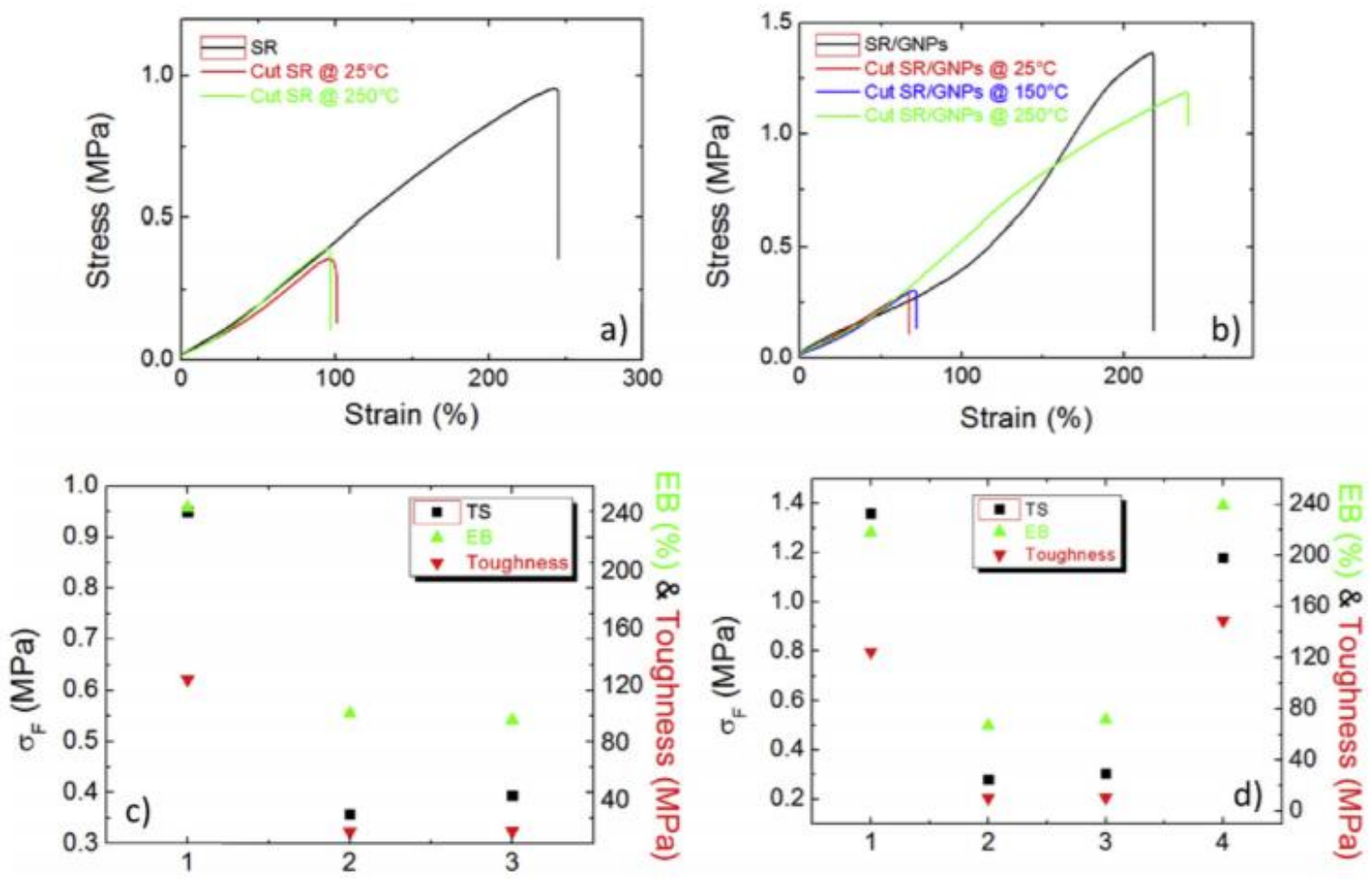

Fig. 3. Stress-strain curves of (a) neat SR, cut SR and cut SR annealed at $250 \mathrm{C}$ and (b) SR/GNP composite, cut composite, cut composite annealed at $150 \mathrm{C}$ and cut composite annealed at $250 \mathrm{C}$, respectively. Panel (c) shows the fracture strength, elongation at break and toughness obtained from the tensile curves of the SR (1), cut SR (2) and cut $\mathrm{SR}$ annealed at $250 \mathrm{C} \mathrm{(3).} \mathrm{Panel} \mathrm{(d)} \mathrm{shows} \mathrm{the} \mathrm{fracture} \mathrm{strength,} \mathrm{elongation} \mathrm{at} \mathrm{break} \mathrm{and} \mathrm{toughness} \mathrm{obtained} \mathrm{from} \mathrm{the}$ tensile curves of the SR/GNP composite (1), cut SR/GNP composite (2), cut SR/GNP composite annealed at 150 C (3) and cut SR/GNP composite annealed at $250 \mathrm{C}(4)$, respectively.
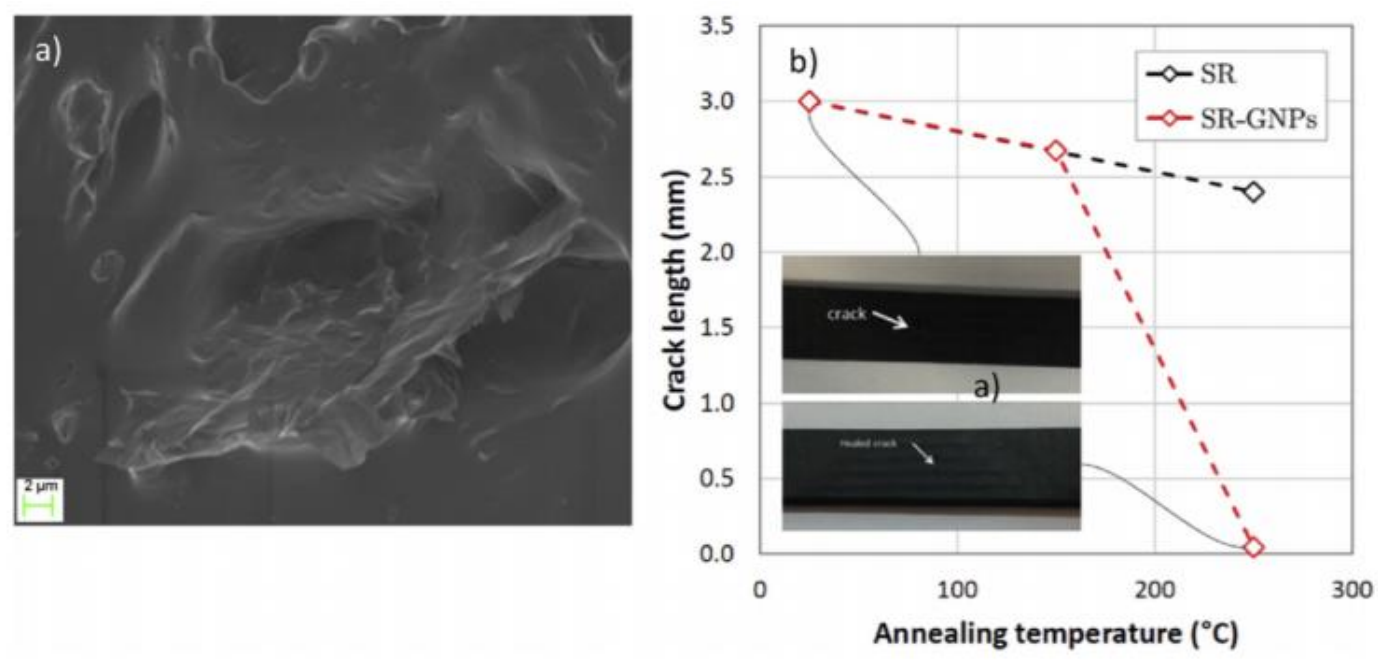
Fig. 4. (a) FESEM image showing the GNP inclusion in the silicone rubber matrix. (b) Crack length vs. annealing temperature of the prepared samples from QFM estimations. The inset shows the optical images of the SR/GNP composite before and after thermal annealing showing the healing of the initial crack length.

Most silicone materials exhibit a series of low-intensity infrared bands in the $\mathrm{OH}$ stretching region. These hydroxyls can be generally found in silicones and are: $\mathrm{SiOH}$, carbinol $(\mathrm{COH})$ and water $(\mathrm{HO}-$ $\mathrm{H})$. These bands occur at $3642(\mathrm{COH}), 3700(\mathrm{HOH})$ and $3763(\mathrm{SiOH}) \mathrm{cm} 1$ [32] and are always present in silicones containing a siloxane chain (-Si-O-Si-). From Fig. $5 \mathrm{a}$ it is evident that in the SR/GNP composite such bands are still visible after the crosslinking while disappearing after annealing at $250 \mathrm{C}$. This finding suggests that free silanol groups are present after the SR/ GNP polycondensation and the curing is not complete. The presence of the band at about $3400 \mathrm{~cm} 1$ appears when hydrogen-bonded hydroxyl species are present. The incomplete curing reflects the behaviour of stress-strain curve at higher elongation (i. e. 150\%) where a decrease of the stress with increasing the strain was observed (Fig. 3b). The non-linear stress-strain behaviour observed in Fig. $3 \mathrm{~b}$ is typical of rubbery elastic materials where the stress and elongation depends on the ratio of the unswollen volume to the swollen volume (vr) (i.e. the polymerized volume fraction) and by the number of the effective number of cross-links (N0) according to the following equation

$$
\left(\rho / M_{c}\right)\left(1-2 M_{c} / M_{n}\right)=N_{0} v_{r}{ }^{1 / 3}
$$

where $r$ is the density of the polymer, $\mathrm{Mc}$ is the number average molecular weight between crosslinks, $\mathrm{Mn}$ is the number average molecular weight of the linear polymer chains before cross-linking [33]. On the contrary the SR system did not give evidence of free silanol groups suggesting a complete curing after polymerization (Fig. 5b) in accordance with the stress increase with the strain as reported in Fig. 3a. 

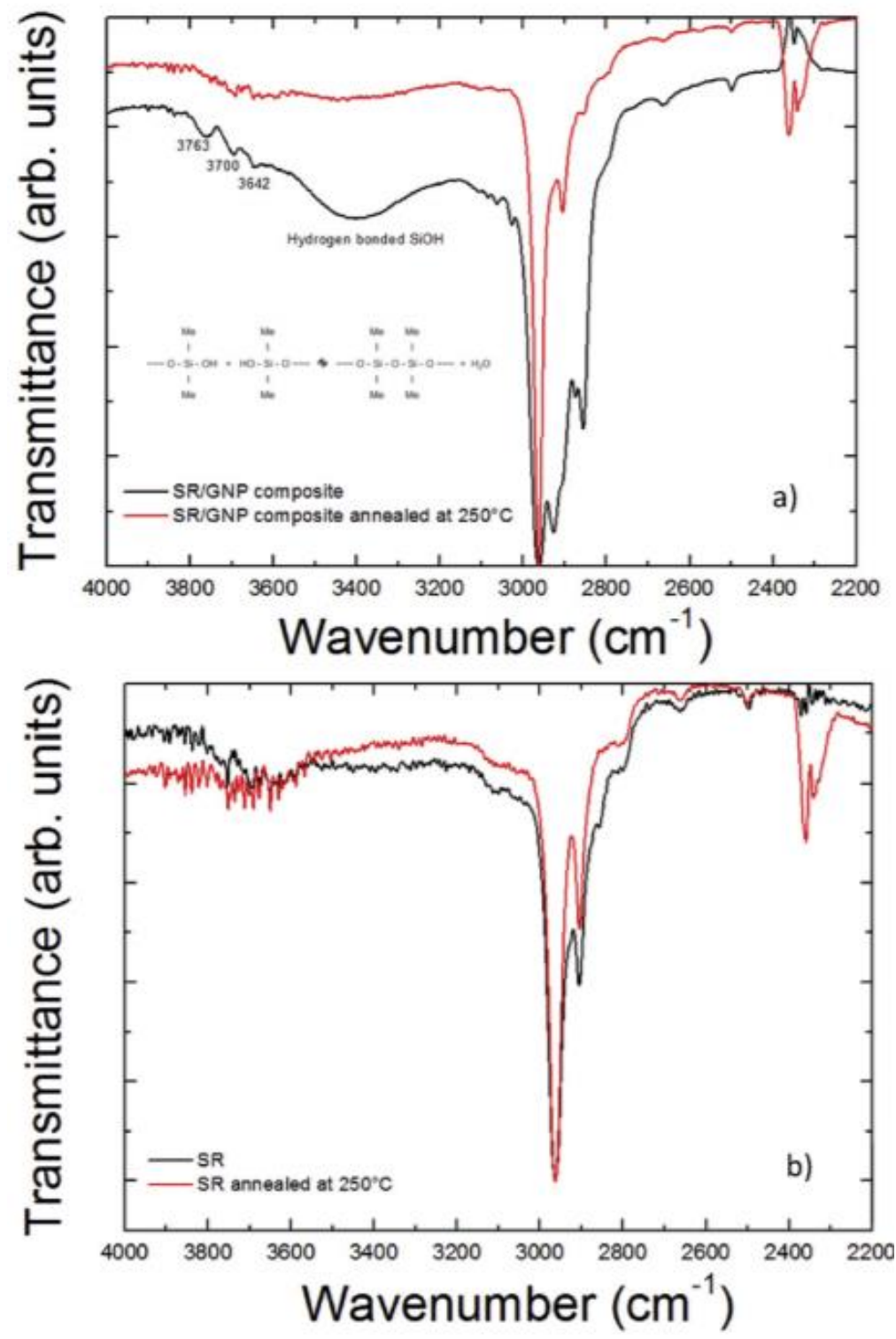

Fig. 5. (a) FTIR spectra of the SR/GNP composite after polymerization (black curve) and annealing at $250 \mathrm{C}$ (red curve). The hydroxyl bands are labeled in the SR/GNP composite after polymerization. The inset in the panel (a) shows the mechanism of linear condensation in silicones. (b) FTIR spectra of the SR sample after polymerization (black curve) and annealing at $250 \mathrm{C}$ (red curve).

Table 1 Crack length vs. annealing temperature of the prepared samples from QFM estimations.

\begin{tabular}{lll}
\hline Healing temperature $\left({ }^{\circ} \mathrm{C}\right)$ & \multicolumn{2}{l}{ Crack length $(\mathrm{mm})$} \\
\cline { 2 - 3 } & SR & SR/GNPs \\
\hline 25 & 3.00 & 3.00 \\
150 & n.a. & 2.67 \\
250 & 2.40 & 0.05 \\
\hline
\end{tabular}

Previous works indicated that the thermal conductivity enhancement achieved in GNP based silicon composites is the highest reported to date in polymer composites [10,34]. In view of previous results [35], the thermal conductivity monotonously increases as the temperature and filler loading increases. Thus in our case the observed healing mechanism is attributed to an increase of the thermal conductivity due to the severe aggregation of GNPs; this result is in contrast with the findings in previous research, in which the thermal conductivity in GNP based composites is only observed at low filler loading $(<20 \%$ vol) [36]. The high healing yield, observed only for the composite, can be 
explained only considering the synergic effect of the high number of inclusions in the agglomeration and the large size of single inclusions. Agglomerated GNPs, work at the macromolecular level of the matrix as a heater and transfer the required energy to the matrix activating the healing of the molecules.

\section{Conclusions}

In summary, novel multifunctional properties of silicone rubber composite system have been reported. GNPs addition to SR demonstrated that such composite can be healed by simple thermal annealing with high yields. The cross-links of polymerized silicone rubber was allowed to be rearranged at $250 \mathrm{C}$, which imparted the composite with the functionality of self-healing. Also, the composite's negative temperature coefficient behaviour suggests new potential as temperature sensors. The remarkable and variable properties of such silicones as well as their wide utilization as polymeric materials suggest that these findings will be broadly applicable.

\section{Acknowledgements}

N.M.P. is supported by the European Research Council (ERC StG Ideas 2011 n. 279985 BIHSNAM, ERC PoC 2015 n. 693670 SILKENE), and by the EU under the FET Graphene Flagship (WP 14 "Polymer nano-composites" n. 696656). S.B.B. was supported by Regione Umbria ("Promozione della ricerca e dell'Innovazione" n. UM12024L002 POR Umbria FSE 2007-2013).

\section{References}

[1] H. Aguilar-Bolados, J. Brasero, M.A. Lopez-Manchado, M. Yazdani-Pedram, High performance natural rubber/thermally reduced graphite oxide nanocomposites by latex technology, Compos. Part B 67 (2014) 449e454.

[2] A. Malas, P. Pal, S. Giri, A. Mandal, C.K. Das, Synthesis and characterizations of modified expanded graphite/emulsion styrene butadiene rubber nanocomposites: mechanical, dynamic mechanical and morphological properties, Compos. Part B 58 (2014) $267 e 274$.

[3] M.H. Flaifel, S.H. Ahmad, A. Hassan, S. Bahri, MaA. Tarawneh, L.-J. Yu, Thermal conductivity and dynamic mechanical analysis of NiZn ferrite nanoparticles filled thermoplastic natural rubber nanocomposite, Compos. Part B 52 (2013) 334e339.

[4] S.M. Shang, L. Gan, M.C.W. Yuen, Improvement of carbon nanotubes dispersion by chitosan salt and its application in silicone rubber, Compos. Sci. Technol. 86 (2013) 129e134.

[5] S.M. Shang, L. Gan, M.C.W. Yuen, S.X. Jiang, M.N. Luo, Carbon nanotubes based high temperature vulcanized silicone rubber nanocomposite with excellent elasticity and electrical properties, Compos. Part A 66 (2014) 135e141.

[6] T. Someya, Y. Kato, T. Sekitani, S. Iba, Y. Noguchi, Y. Murase, H. Kawaguchi, T. Sakurai, Conformable, flexible, large-area networks of pressure and thermal sensors with organic transistor active matrixes, Proc. Natl. Acad. Sci. 102 (2005) 12321 e12325.

[7] K. Takei, T. Takahashi, J.C. Ho, H. Ko, A.G. Gillies, P.W. Leu, R.S. Fearing, A. Javey, Nanowire active-matrix circuitry for low-voltage macroscale artificial skin, Nat. Mater 9 (2010) 821e826.

[8] H. Yousef, M. Boukallel, K. Althoefer, Tactile sensing for dexterous in-hand manipulation in robotics-a review, Sens. Actuat. A 167 (2011) 171 e187. 
[9] S. Shang, L. Gan, M. Chun-wah Yuen, S.-X. Jiang, N.M. Luo, Carbon nanotubes based high temperature vulcanized silicone rubber nanocomposite with excellent elasticity and electrical properties, Compos. Part A 66 (2014) 135e141.

[10] M.A. Raza, A. Westwood, A. Brown, N. Hondow, C. Stirling, Characterisation of graphite nanoplatelets and the physical properties of graphite nanoplatelet/ silicone composites for thermal interface applications, Carbon 49 (2011) 4269e4279.

[11] J. Zhang, S. Feng, X. Wang, DC current voltage characteristics of silicone rubber filled with conductive carbon black, J. Appl. Pol. Sci. 94 (2004) 587e592.

[12] D. Kong, L.T. Le, Y. Li, J.L. Zunino, W. Lee, Temperature-dependent electrical properties of graphene inkjet-printed on flexible materials, Langmuir 28 (2012) 13467e13472.

[13] Y. Chen, A.M. Kushner, G.A. Williams, Z. Guan, Multiphase design of autonomic self-healing thermoplastic elastomers, Nat. Chem. 4 (2012) 467e472.

[14] S.R. White, N.R. Sottos, P.H. Geubelle, J.S. Moore, M.R. Kessler, S.R. Sriram, E.N. Brown, S. Viswanathan, Autonomic healing of polymer composites, Nature 409 (2001) 794e797.

[15] K. Kratz, A. Narasimhan, R. Tangirala, S. Moon, R. Revanur, S. Kundu, H.S. Kim, A.J. Crosby, T.P. Russell, T. Emrick, G. Kolmakov, A.C. Balazs, Probing and repairing damaged surfaces with nanoparticle-containing microcapsules, Nat. Nanotechnol. 7 (2012) 87e90.

[16] Y. Zhao, W. Zhang, L.P. Liao, S.J. Wang, W.J. Li, Self-healing coatings containing microcapsule, Appl. Surf. Sci. 258 (2012) 1915e1918.

[17] P. Cordier, F. Tournilhac, C. Soulie-Ziakovic, L. Leibler, Self-healing and ther- moreversible rubber from supramolecular assembly, Nature 451 (2008) 977e980.

[18] C.-H. Li, C. Wang, C. Keplinger, J.-L. Zuo, L. Jin, Y. Sun, P. Zheng, Y. Cao, F. Lissel, C. Linder, X.-Z. You, Z. Bao, A highly stretchable autonomous self-healing elastomer, Nat. Chem. 8 (2016) $618 \mathrm{e} 624$.

[19] X. Tian, S. Shaw, K.R. Lind, L. Cademartiri, Thermal processing of silicones for green, scalable, and healable superhydrophobic coatings, Adv. Mater. 28 (2016) 3677 e3682.

[20] P. Zheng, T.J. McCarthy, A surprise from 1954: siloxane equilibration is a simple, robust, and obvious polymer self-healing mechanism, J. Am. Chem. Soc. 134 (2012) 2024e2027.

[21] Z.H. Wang, Y.L. Lu, J. Liu, Z.M. Dang, L.Q. Zhang, W. Wang, Preparation of nanoalumina/EPDM composites with good performance in thermal conductivity and mechanical properties, Polym. Adv. Technol. 22 (2011) $2302 \mathrm{e} 2310$.

[22] L.Q. Zhang, S.M. Wu, H.P. Geng, X.B. Ma, Q. Leng, Y.X. Feng, China Synth. Rubber Ind. 21 (1998), 207-.

[23] V. Causin, C. Marega, A. Marigo, G. Ferrara, A. Ferraro, Morphological and structural characterization of polypropylene/conductive graphite nanocomposites, Eur. Polym. J. 42 (2006) 3153 e3161.

[24] S. Ganguli, A.K. Roy, D.P. Anderson, Improved thermal conductivity for chemically functionalized exfoliated graphite/epoxy composites, Carbon 46 (2008) 806e817.

[25] Y.L. Wang, Y.A. Hu, L. Chen, X.L. Gong, W.Q. Jiang, P.Q. Zhang, Effects of rubber/ magnetic particle interactions on the performance of magnetorheological elastomers, Polym. Test. 25 (2006) $262 \mathrm{e} 267$. 
[26] F. Zhuge, B. Hu, C. He, X. Zhou, Z. Liu, R. Li, Mechanism of nonvolatile resistive switching in graphene oxide thin films, Carbon 49 (2011) $3796 e 3802$.

[27] J. Kang, J. Ryu, G. Han, J. Choi, W. Yoon, B. Hahn, J. Kim, C. Ahn, J. Choi, D. Park, LaNiO3 conducting particle dispersed NiMn2O4 nanocomposite NTC thermistor thick films by aerosol deposition, J. Alloys Compd. 534 (2012) 70e73.

[28] N.M. Pugno, R.S. Ruoff, Quantized fracture mechanics, Philos. Mag. 27 (2004) 2829e2845.

[29] L. Valentini, S. Bittolo Bon, M.A. Lopez-Manchado, R. Verdejo, L. Pappalardo, A. Bolognini, A. Alvino, S. Borsini, A. Berardo, N.M. Pugno, Synergistic effect of graphene nanoplatelets and carbon black in multifunctional EPDM nanocomposites, Comp. Sci. Technol. 128 (2016) 123e130.

[30] R. Ansari, S. Ajori, B. Motevalli, Mechanical properties of defective singlelayered graphene sheets via molecular dynamics simulation, Superlattices Microstruct. 51 (2012) 274e289.

[31] H.P. Xiang, M.Z. Rong, M.Q. Zhang, Self-healing, reshaping, and recycling of vulcanized chloroprene rubber: a case study of multitask cyclic utilization of cross-linked polymer, ACS Sustain. Chem. Eng. 4 (2016) 2715e2724.

[32] G.W. Griffith, Quantitation of silanol in silicones by FTIRspectroscopy, Ind. Eng. Chem. Prod. Res. Dev. 23 (1984) 590e593.

[33] K.S. Anseth, C.N. Bowman, L. Brannon-Peppas, Mechanical properties of hydrogels and their experimental determination, Biomaterials 17 (2012) 1647e1657.

[34] K.M.F. Shahil, A.A. Balandin, Thermal properties of graphene and multilayer graphene: applications in thermal interface materials, Solid State Comm. 152 (2012) 1331e1340.

[35] X. Huang, C. Zhi, P. Jiang, Toward effective synergetic effects from graphene nanoplatelets and carbon nanotubes on thermal conductivity of ultrahigh volume fraction nanocarbon epoxy composites, J. Phys. Chem. C 116 (2012) 23812e23820.

[36] A.P. Yu, P. Ramesh, X.B. Sun, E. Bekyarova, M.E. Itkis, R.C. Haddon, Enhanced thermal conductivity in a hybrid graphite nanoplatelet-carbon nanotube filler for epoxy composites, Adv. Mater. 20 (2008) 4740e4744. 